\title{
Recommendations for Operation, Measurement, Reporting and Application of Pediatric Lung Ultrasound: Chinese Experts Consensus
}

\author{
Pediatric Ultrasound Committee of Ultrasound Branch of Chinese Medical Education Association; Ultrasound \\ Committee of Chinese Pediatric Society, Chinese Medical Association; Weiling Chen, MD ${ }^{a}, B_{e i}$ Xia, MD ${ }^{a, *}$, Lan \\ Wang, MD ${ }^{b, *}$, Lixue Yin, $M D^{c, *}$

\begin{abstract}
${ }^{a}$ Department of Ultrasound, Shenzhen Children's Hospital, Shenzhen, China; ${ }^{b}$ Department of Pediatric Ultrasound, the First Hospital of Jilin University, Changchun, China; ${ }^{c}$ Institute of Ultrasound in Medicine, Sichuan People's Hospital, Sichuan Academy of Medical Sciences, Chengdu, China

Received July 05, 2020; revision received July 28, 2020; accepted July 29, 2020.
\end{abstract}

Advanced Ultrasound in Diagnosis and Therapy 2021;01:001-011

DOI: 10.37015/AUDT.2021.200056

\section{Introduction}

Based on changes in the ratio of air to water or lung tissue, pathological mechanisms, and acoustic phenomena, lung ultrasound is no longer limited to the diagnosis of pleural effusions. Lung ultrasound has been used for the diagnosis of lung parenchymal diseases in critical care settings and surgeries to reduce unnecessary radiation exposures.

It is necessary to further understand and standardize operational methods to improve the diagnostic ability of pediatric lung diseases. This article focuses on the operation, measurement, and reporting of lung ultrasound, with the purpose of promoting the clinical application of pediatric lung ultrasound.

\section{Access to Clinical Evidence}

Publications from January 2000 to January 2020 were searched on PubMed, MEDLINE, CBMDisc (Chinese Biomedical Literature Database), and CNKI (China Knowledge Network Full-text Database). A combination of the following medical keywords (MeSH) was used for searching: "ultrasonography", "chest sonography", "lung ultrasound", "diagnostic imaging", "respiratory tract diseases", "Pneumonia", "Pulmonary embolism", "pneumothorax", "cardiogenic pulmonary edema", "non-cardiogenic pulmonary edema", "lung tumor", "atelectasis", "interstitial lung disease", "pulmonary fibrosis", "pleural effusion", etc.

We validated the references and selected the articles based on the relevance and level of evidence. The selected references included meta-analysis, casecontrol studies, randomized-control studies, cohort studies, guidelines, and expert consensus. Finally, 203 publication were included in the process of creating the recommendations.

\section{Evidence Level and Recommendation}

Lung ultrasound has been used in neonates and children for more than 10 years [1]. Few articles have published in regard to expert consensus on lung ultrasound in neonates and neocoronavirus-19 lung infections [2, 3]. Based on the practical experience

\footnotetext{
"Corresponding author: Bei Xia, Department of Ultrasound, Shenzhen Children's Hospital, Yitian Road, Futian District, Shenzhen 518038, China.e-mail: xiabeimd@qq.com. Lan Wang, Department of Ultrasound, the First Bethune Hospital of Jilin University, No.1 Xinmin District, Changchun 130021, China.e-mail:15943058071@163.com. Lixue Yin, Institute of Ultrasound in Medicine, Sichuan People's Hospital, Sichuan Academy of Medical Sciences, 2nd West Section, 1st Roundway, Chengdu 610071, China. e-mail: yinlixue_ cardiac@163.com
} 
of every medical center and the discussion in several national academic conferences, the Children's Ultrasound Group of the Ultrasound Professional Committee of the China Medical Education Association was established in 2018 to discuss and formulate this expert consensus. This consensus document will continue to be improved according to feedback in the future. The recommendation level of this consensus is shown in Table $1[4,5]$.

Table 1 Recommended level

\begin{tabular}{ll}
\hline Recommendation strength & Quality of evidence for recommendation \\
\hline A: Strong recommendation for the statement & I : Data come from many randomized trials with clinical outcomes and/or meta-analyses \\
B: Moderate recommendation for the statement & II : Data come from individual randomized trials and/or large non-randomized trials \\
C: Optional recommendation for the statement & III: Expert opinion and/or data from small studies, retrospective studies, or case reports \\
\hline
\end{tabular}

\section{Indications}

Lung ultrasound has high accuracy in the diagnosis of lung consolidation, lung pleural effusion, and pneumothorax. Therefore, it is useful for diagnosing severe trauma, respiratory failure, and shock for pediatric patients $[4,6]$. The changes in the ratio of air to fluid in the lung glandular lobule result in different images of pulmonary interstitial lesions, pulmonary edema, and lung consolidation [7]. Lung ultrasound can be used for the screening of pneumonia, differential diagnosis of cyanosis, hypotension, and severe infectious diseases as well [8]. In open surgery, lung ultrasound can also be utilized in patients with unstable cardiopulmonary conditions or suspected pneumothorax $[9,10]$. For neoplastic diseases, lung ultrasound can identify nodules and metastatic lesions in the pleural cavity and subpleural areas [11]. In interventional surgery, guiding the biopsy of sub-pleural lung parenchyma or lung nodules in real time is another application of lung ultrasound [12]. Sine there are no contraindications for pediatric lung ultrasound, it could be safely carried out in pediatric patient population $[13,14]$.

\section{Equipment and Methods}

\section{Instrument}

Color Doppler ultrasound for lung exam is best performed with linear transducers for chest wall, convex or micro-convex transducers for subcostal scanning, and phased-array transducers for parasternal and suprasternal scanning. With enough penetration, the frequency should be set as high as possible. Convex transducer $(3-5 \mathrm{MHz})$ is used for fat or deep lesions, and higher frequency linear transducer $(7.5-12 \mathrm{MHz})$ is used for pleural or sub-pleural lesions. It is better to use linear transducer $(>7 \mathrm{MHz})$ in neonate. Convex or phased-array transducer $(3-7.5 \mathrm{MHz})$ is used for mediastina scanning.

\section{Regulation}

Clinicians should aware that ultrasound wave can create mechanical forces causing pressure changes and release of various reactive molecules, and gas-containing organs (e.g., lung) are susceptible to the effects of acoustic cavitation. Following the principle of as low as reasonably achievable (ALARA), the potential benefits and risks should be considered in each examination. Lowering the output power to $\mathrm{MI}<0.6$, increasing the gain, adjusting the TCG curve, and using harmonics, compound imaging, and logiqview to display all lesions could help reduce the risk of mechanical tissue injury from lung ultrasound. Moreover, the imaging depth should be at least 3 to 4 times the distance between the A-lines, and the color Doppler should be adjusted to a scale of $5-20 \mathrm{~cm} / \mathrm{s}$.

\section{Imaging mode}

Pediatric lung ultrasound imaging modes include twodimensional imaging (level of evidence A), color Doppler (level of evidence A), contrast enhance ultrasound [1517] (level of evidence B), and elastography [18,19] (level of evidence C). Color Doppler ultrasound is mainly used to identify the presence or lack of blood flow and arteriovenous fistula, while contrast enhanced ultrasound is used to identify tumor, abscess, contusion, and laceration.

\section{Qualifications and Skills}

\section{Training}

The operator should understand the basic knowledge of the anatomy and physiology of the lungs, thoracic cavity, and mediastinum. After the mandatory training, the operator should be able to accurately identify basic normal and abnormal sonograms, correctly apply ultrasound-guided thoracentesis [20-22], and perform at least 30 cases of lung and pleural effusion under the guidance of professional physicians, including at least 20 positive cases. 


\section{Operation method}

Position Supine and lateral positions are applied in newborns and infants. Children are examined in a sitting position, and severely ill children were placed in supine or semi-recumbent positions (Fig. 1) (Recommended level A). Ultrasound examination should be conducted in a quiet environment.
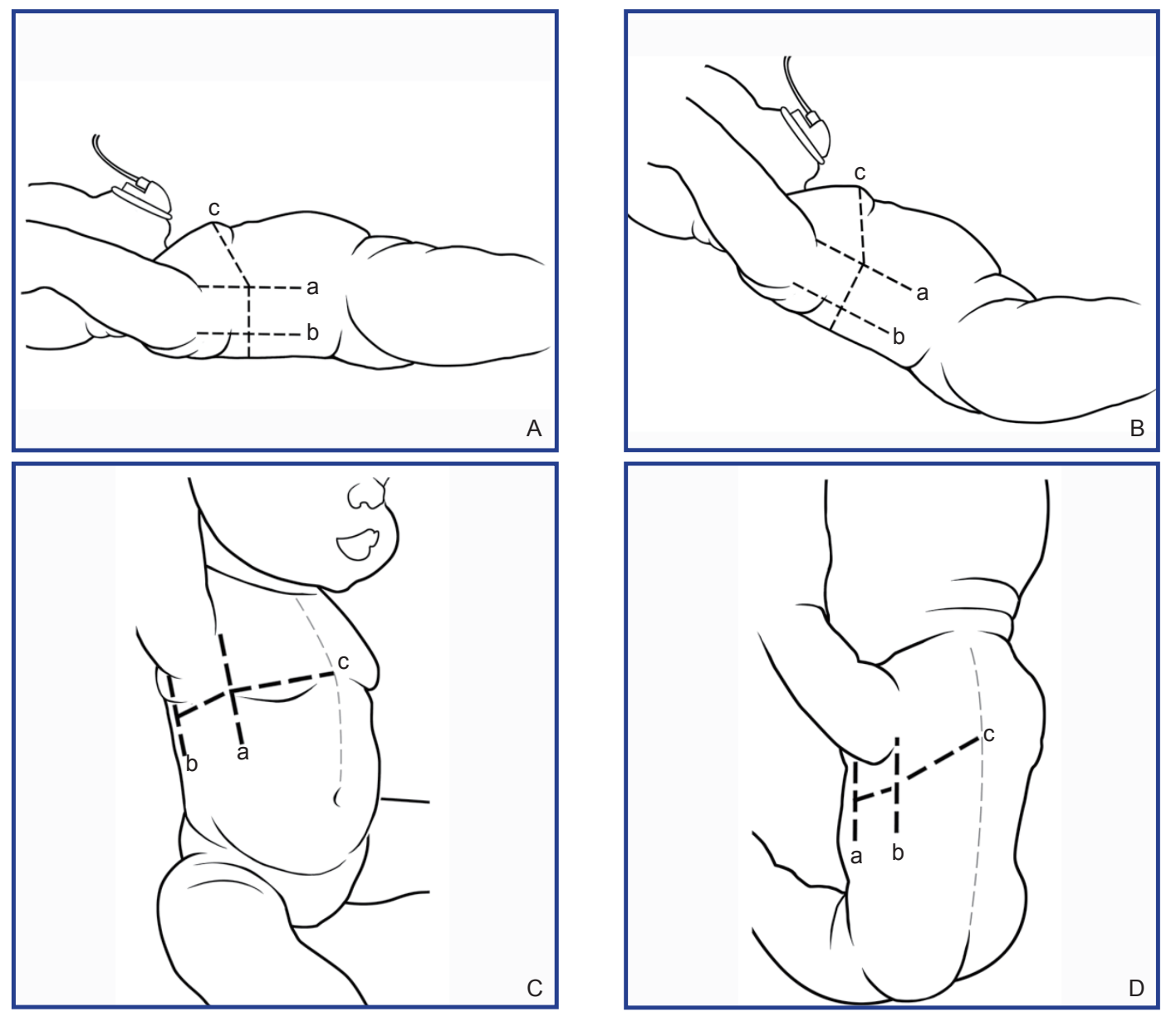

Figure 1 Position and body surface partition. (A) Supine position; (B) Semi-recumbent position; (C $\sim$ D) Sitting position. a, anterior axillary line; b, posterior axillary line; $c$, the connecting line between the two nipples. Named by L/R-A/L/P-U/L method: the chest surface is divided into left (L) and right (R), which are divided into anterior (A), lateral (L) and posterior (P) zones by four lines, and the above three parts are divided into upper (U) and lower (L) parts by horizontal line connecting nipples.

The body surface partitions of lung scan are shown in Figure 1 (Recommended level A). The areas need to be examined including trans-thoracic wall, subcostal, parasternal, and suprasternal. Subcostal scan shows the image of the lung diaphragmatic surface, and scan through the superior sternal fossa and parasternal scan can show an image of the mediastinal surface. Most critically ill children require a comprehensive evaluation of cardiac and lung ultrasound. Transthoracic scans use the longitudinal section to move the probe horizontally and the intercostal transverse section to move up and down and swing the probe. The operation method is shown in Figure 2 (Recommended level A).

\section{Basic image identification}

A-Line (Recommended level A): It is a complete reflection (repetition artifacts) formed by a highly reflective surface. It is found in normal lungs, emphysema, pneumothorax, and intestinal gas (e.g., intestinal canal in diaphragmatic hernia) (Fig. 3).

B-Line (Recommended level A): It is a ring-down artifact generated by mixing of small-diameter gas with surrounding water and tissues. The exudation and hyperplasia of alveoli and interstitial tissue mixes with gas to form the B-line (Fig. 4), which is also happen in the mixtures of intestinal gas and fecal fluid. B-Line is a pathological phenomenon caused by the increase in the proportion of fluid or exudations from tissue in the lungs ("wet changes" of the lungs). B-Line occurs in diseases such as pneumonia, cardiogenic and non-cardiogenic pulmonary edema, respiratory distress syndrome, lung trauma, and pulmonary interstitial hyperplasia, etc. 

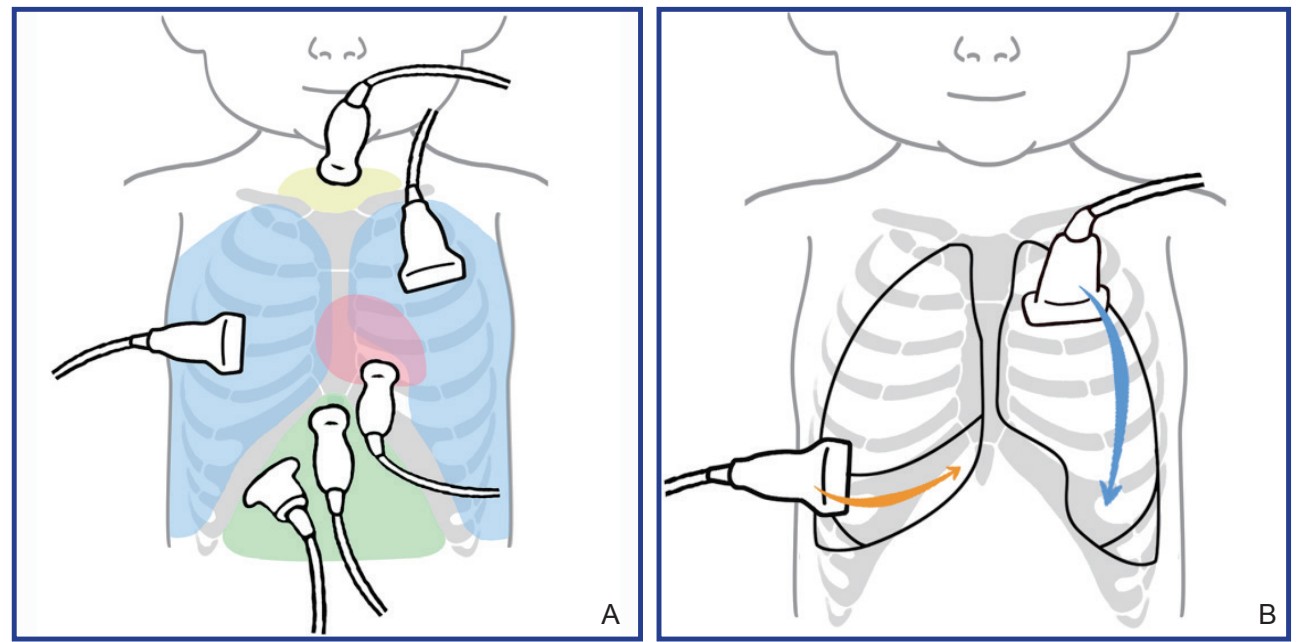

Figure 2 Schematic diagram of the body surface partition of the lung scan. (A) Transthoracic lung surface scan (blue), subcostal scan (green), parasternal precordial area scan (red), and trans sternal scan (yellow); (B) Transthoracic scan technique: The probe is vertical to the chest wall surface, moving and swinging the probe, the longitudinal section moves laterally (orange arrow), and the transverse section moves up and down (blue arrow).
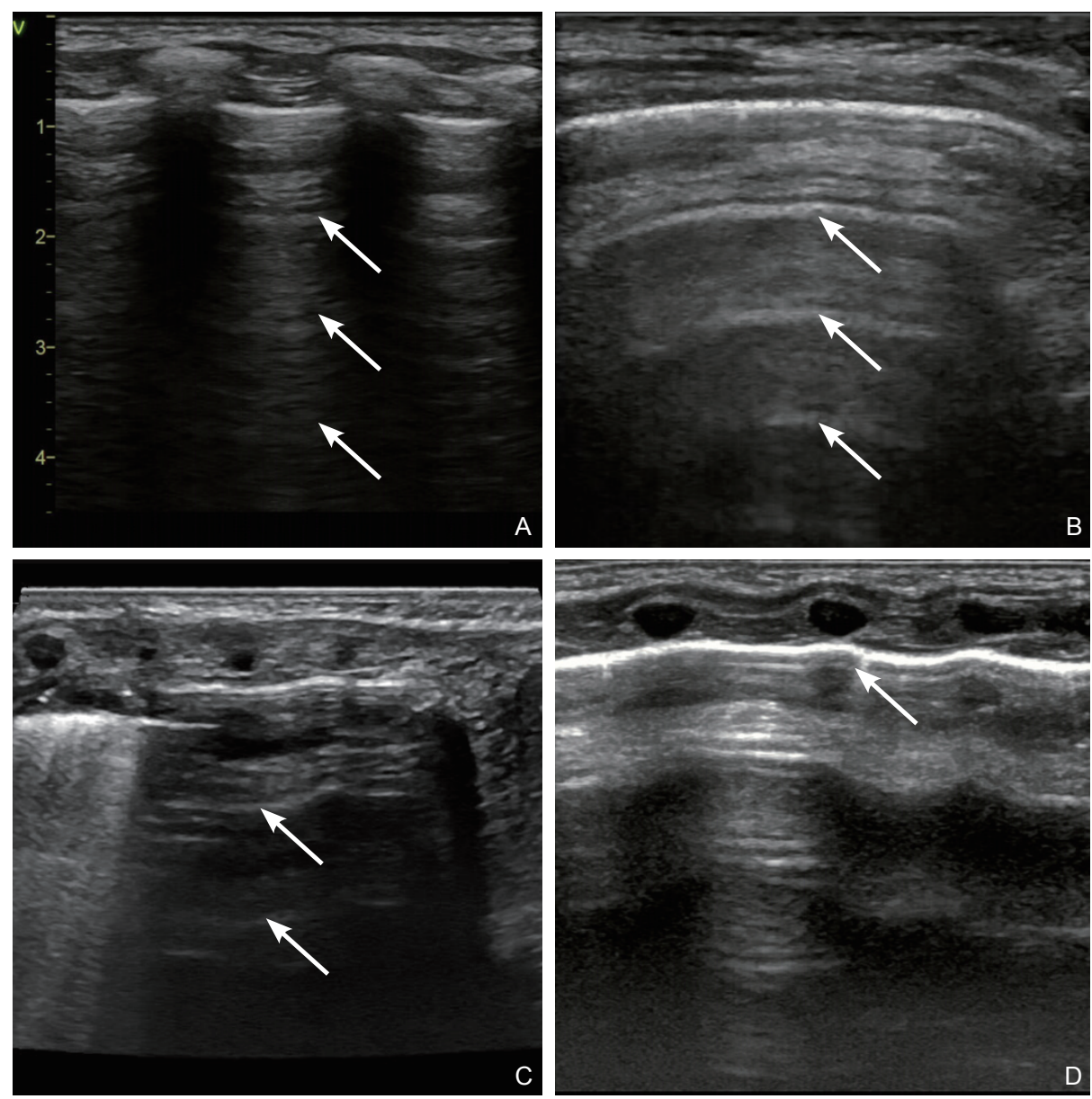

Figure 3 A-lines. $(\mathrm{A} \sim \mathrm{B})$ Normal pulmonary sonogram; $(\mathrm{C} \sim \mathrm{D})$ pneumothorax. (A) Transthoracic transverse section, the arrow is shown as A-line; (B) Transthoracic longitudinal section, the arrow is shown as A-line; (C) A-line of gas formation in pleural cavity (arrow); (D) Sporadic B line (arrow) indicates that A-line originates from the lung.

Pulmonary consolidation (Recommended level A): Pulmonary consolidation is a non-gas expansion of alveoli, occurs in inflammation, atelectasis, pulmonary embolism, tumors and congenital malformations. The manifestation of diffuse and widely distributed lung consolidation does not have specific pathological 
significance (Fig. 5), because many diseases can present the similar lung consolidation image. Abnormal "pleural line" is not formed by pleural tissue but a small consolidation caused by increased fluid in the sub-pleural interstitial lung or alveoli.
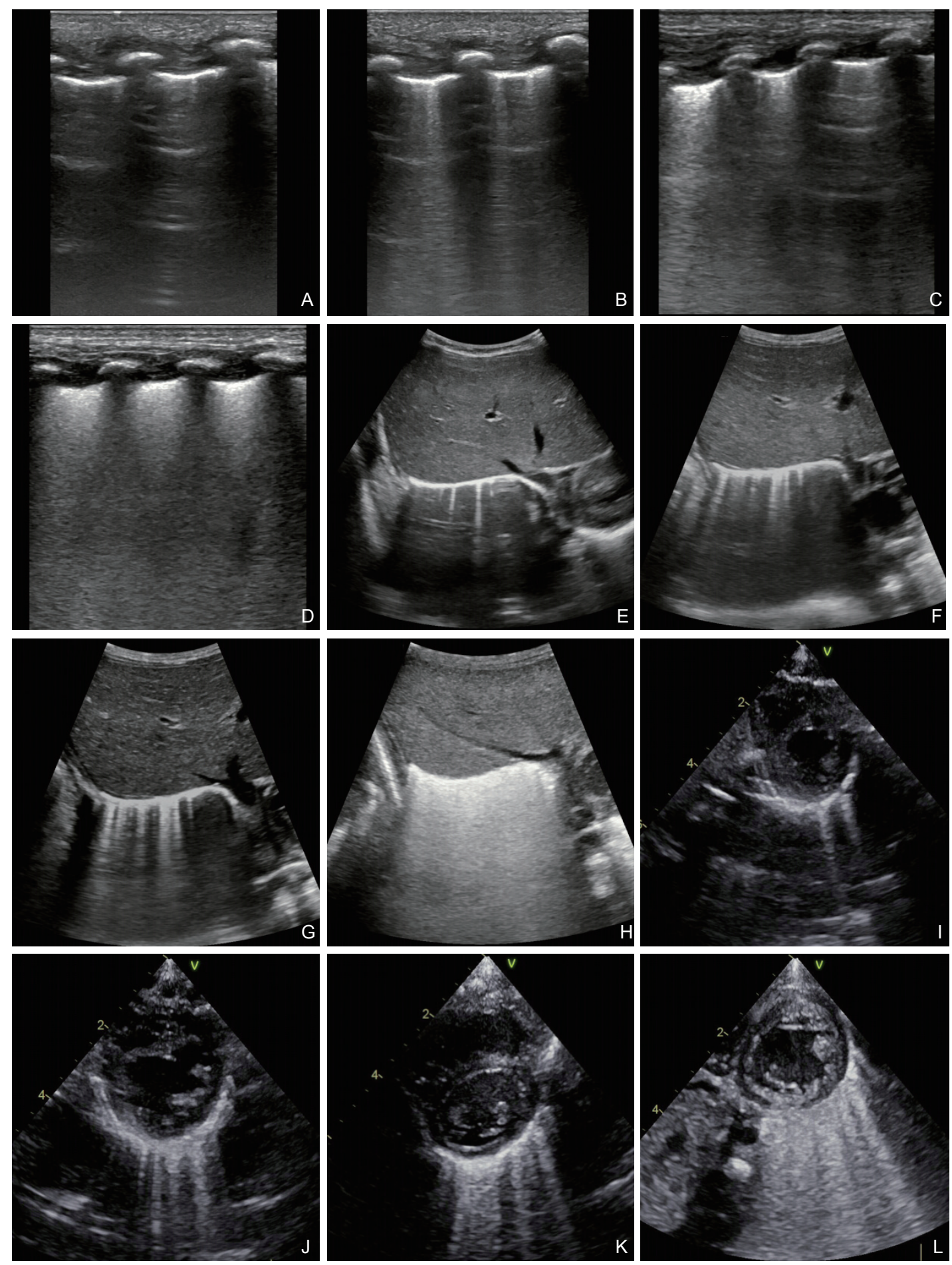

Figure 4 B-lines. (A D) B-line scan of Transthoracic lung surface; (E H) B-line scan of subcostal; (I L) B-line scan of parasternal precordial area. Scattered B-lines, sparse B-lines, dense B-lines and fused B-lines are displayed respectively.

Lung abscess (Recommended level A): It is a local anechoic or hypoechoic of lung tissue due to infection and necrosis, and color Doppler flow image shows no blood flow within mass [23] (Fig. 6). 

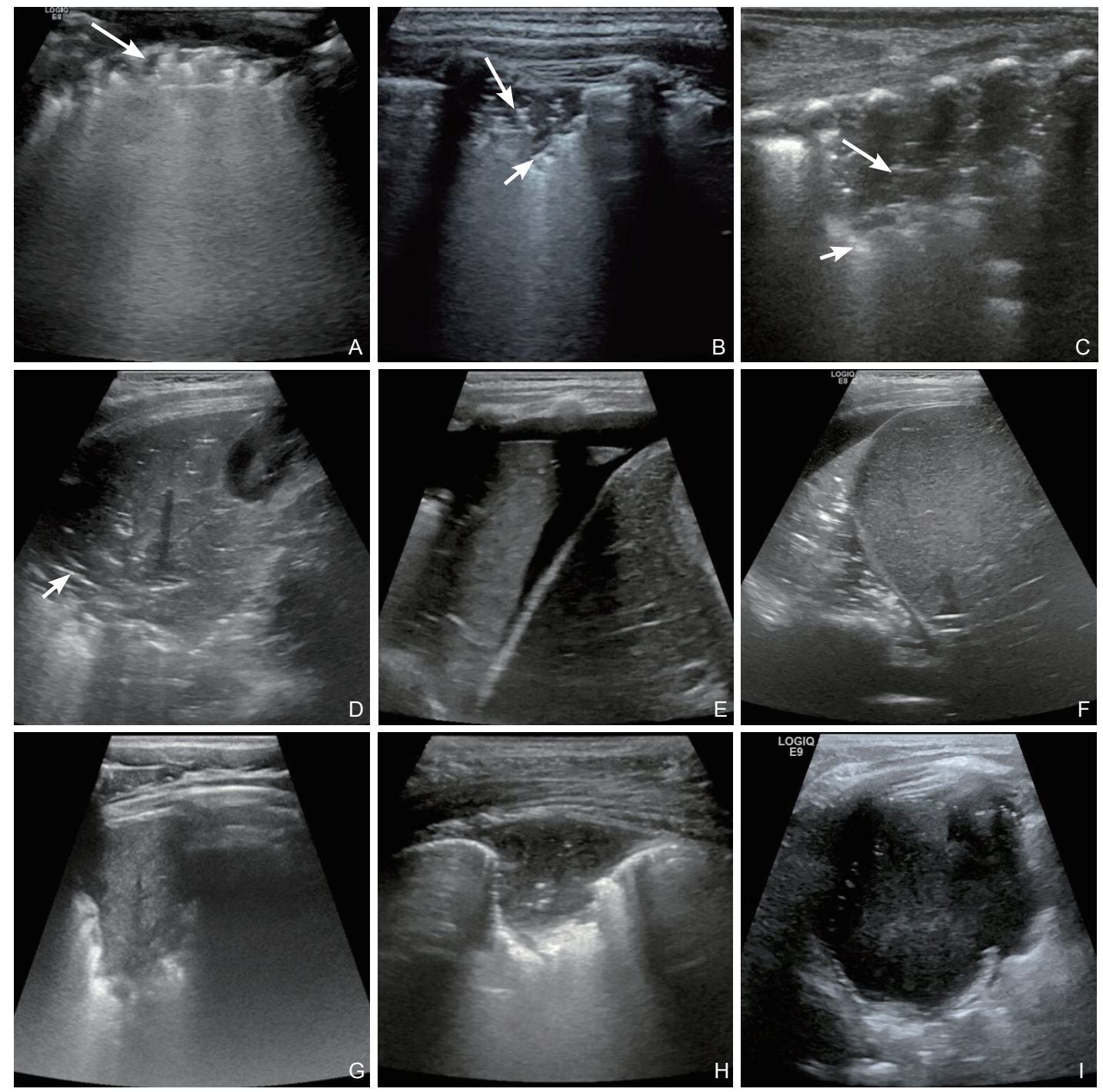

Figure 5 Lung consolidation sonograms. (A) Small fragment-like consolidation scattered under the pleural line (long arrow); (B C) Pulmonary consolidation was accompanied by bronchial inflation echo (long arrow) and peripheral pulmonary B-line echo (short arrow); (D) Local atelectasis with pleural effusion; (E) Pulmonary consolidation after ECMO; (F) Pulmonary embolism showed wedge-shaped consolidation; (G) Necrotizing granuloma of lung; (H) Consolidation of lung with fluid bronchogram (arrow); (I) Pleuropulmonary blastoma.
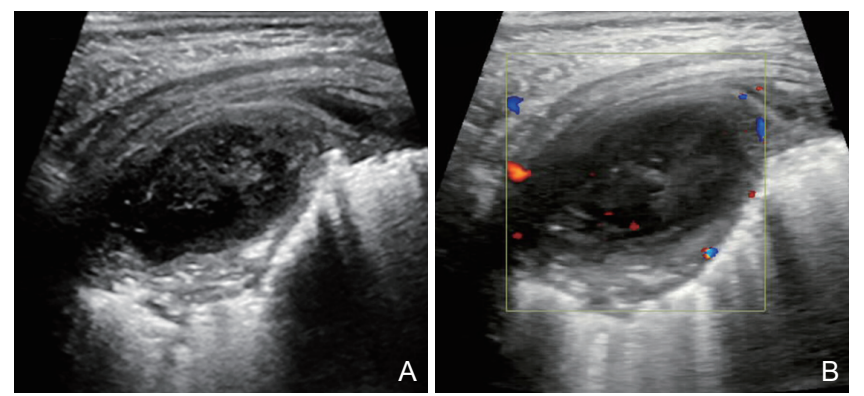

Figure 6 Pulmonary abscess sonograms. (A) Consolidated lung tissue showed a liquid dark area echo; (B) There was no blood flow signal in CDFI.

Pneumothorax (Recommended level A): Pneumothorax presents with a fixed A-line does not move with the breath, and there is no B-line displays. A small amount of pneumothorax can be found in the "lung point" between the fixed A-lines when the moving with the breath [4]. Traumatic pneumothorax is usually combined with pleural effusion (hemorrhage) and lung contusion.

Pulmonary infarction (Recommended level B): The sonogram shows pulmonary consolidation without blood flow. Contrast enhanced ultrasound shows no enhancement [24].

Pleural effusion (Recommended level A): Ultrasound images of pleural effusion have various manifestations due to different causes.

Interventional ultrasound (Recommended level A): Ultrasound-guided thoracentesis biopsy, aspiration, or catheter drainage increase the accuracy and safety of clinical procedures in pediatric [12].

\section{Measurement}

Quantification of pleural effusion (Recommended 
level A): The amount of pleural effusion in pediatrics is semi-quantitative. While the patients lie in the supine position, the range of fluid dark areas will be evaluated at intercostal level in the anterior axillary line, midaxillary line, and posterior axillary line or subscapular line. Healthcare providers should measure the maximum depth from the pleural wall layer to the visceral layer perpendicular and further to the chest wall.

Line semi-quantitative counting (Recommended level C): If necessary, use B-line counting method to evaluate the degree of increase in lung interstitial or lung lobular water [25-32].

Lung consolidation (Recommended level A): It is recommended to measure the location, size, air and fluid bronchograms, and color Doppler blood flow.

\section{Application Recommendations}

\section{Pleural effusion (Level A I)}

Due to the different composition of the effusion, such as transudate, pus, blood, and chyle, etc., pleural effusion usually presents with varies images including the thickening of the pleura, the presence or absence of multiple separations, and wrapped effusions [33,34]. Ultrasound examination is used to assess the severity, evaluate the dynamic changes of the lesion, and guide the puncture [1].
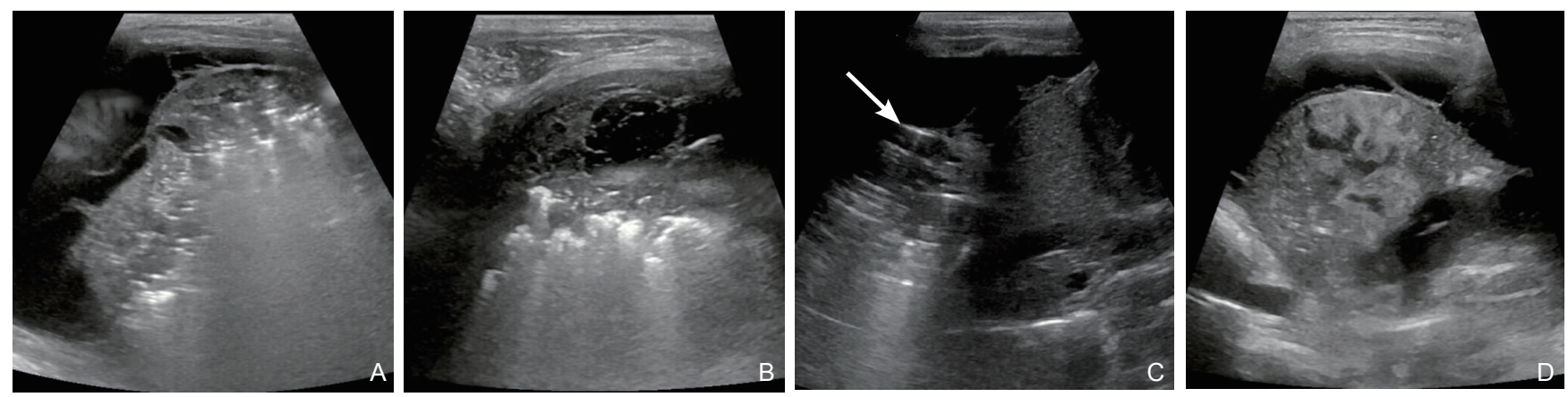

Figure 7 Pneumonia sonograms. (A) Large patchy pulmonary consolidation with massive pleural effusion; (B) The pleura is thickened, and there are effusions in the pleural cavity, with dense point echoes in the effusions; (C) Bronchial pleural fistula, the arrow shows the gas at the fistula; (D) Pulmonary abscess.

\section{Atelectasis (Level A I)}

There are two types of atelectasis: compressive atelectasis and obstructive atelectasis. Compressive atelectasis is caused by extra-pulmonary compression, which is more commonly seen in pediatric patients with pleural effusions. The typical ultrasound presentation is lung consolidation connecting to the hilum combined with removable bronchial gas. Obstructive atelectasis is caused by bronchial obstruction (e.g., inflammation, hematoma, tumor, and foreign body, etc.), and images will show that the lungs are evenly parenchymal. The

\section{Severe pneumonia (Level A I )}

Pneumonia is an inflammatory disease of the terminal airway, alveoli, and the surrounding pulmonary interstitial tissue. Because of different etiology and pathological type, the severity and complications of pneumonia varies, which are reflected by different imaging manifestations. Common ultrasound manifestations are A-lines (survival area), B-lines, and lung consolidations. Color Doppler usually shows localized rich blood flow within the consolidation [35-37]. Ultrasound examination focuses on identifying the size, range and symmetry of the B-line, and consolidation on both lungs. Mild pneumonia may have no abnormal ultrasound findings, or only the B-lines show up under the pleura. Severe pneumonia patients have obvious lung consolidation and may present with pulmonary complications, such as pleurisy, pleural effusion, empyema, bronchopleural fistula, and lung abscess, etc. (Fig. 7), and those are the main indications for performing ultrasound examination in children with pneumonia. Clinicians should also pay particular attention to the extra-pulmonary complications of pediatric severe pneumonia, e.g., myocarditis, pericardial effusion, kidney injury, abdominal compartment syndrome [38]. In addition, lung ultrasound is helpful to show the dynamic changes of $\mathrm{B}$-lines and consolidations during the recovery period [39]. bronchus is full of gas or fluids but does not move, and rarely shows obstructive pathological masses (Fig. 8) [40]. Color Doppler often shows branched blood flow signals in local lung tissue [4].

\section{Cardiogenic pulmonary edema (Level A I)}

Pulmonary edema is caused by various causes of abnormal fluid quickly extravasates from capillaries and accumulates in the alveoli and/or interstitial lung, forming alveolar pulmonary edema and/or interstitial pulmonary edema, which could further cause severe 
respiratory failure. Based on different etiology, there are two types of pulmonary edema: cardiogenic pulmonary edema and non-cardiogenic pulmonary edema. Cardiac pulmonary edema is characterized by bilateral, symmetric, gravity-dependent distribution of B-lines [27], and the distribution is related to the severity of the disease.
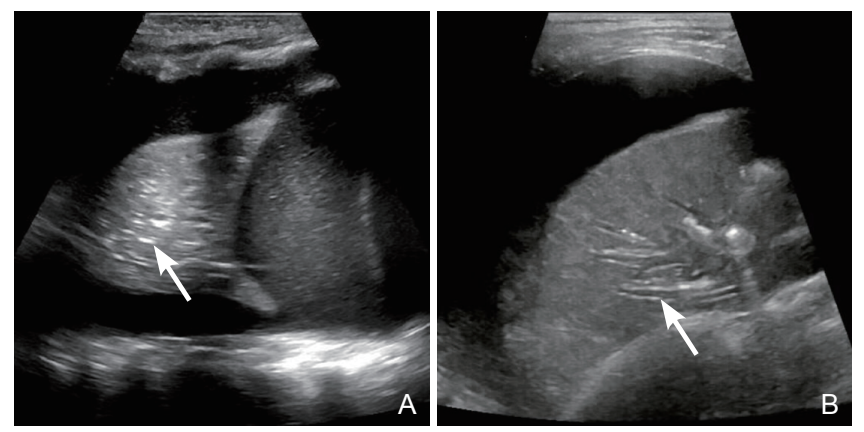

Figure 8 Atelectasis sonograms. (A) Air bronchogram (arrow); (B) Fluid bronchogram (arrow).

\section{Acute respiratory distress syndrome (Level B I)}

Acute respiratory distress syndrome (ARDS) is a diffuse injury of pulmonary capillaries resulting in increased capillary permeability and decreased lung compliance, followed by alveolar collapse, and ventilation-perfusion imbalance. The basic pathology is diffuse alveolar exudation and lung consolidation.

Ultrasound findings of ARDS often consist of small or massive consolidations under the pleura of the anterior chest wall, thickened hyperechoic area on the lung surface, weakened or disappeared lung sliding signs, and unevenly distributed B-lines [4,41]. For those who improves after treatment, the diffused B-lines will change to localized B-lines, and the lungs will return to the A-lines eventually.

\section{Pulmonary trauma (Level A I)}

Pediatric lung injury is most commonly from blunt trauma, which usually resulting in hemothorax, pneumothorax, lung contusion, lung laceration, pericardial effusion, and rupture of the heart [42]. Lung contusion can lead to potential ARDS. Lung ultrasound can quickly assess pleural effusion, pneumothorax and B-line distribution, and lung consolidation (Fig. 9). Since chest trauma is usually combined with abdominal injury, using rapid ultrasound is helpful in emergency settings to guide further treatment $[43,44]$.
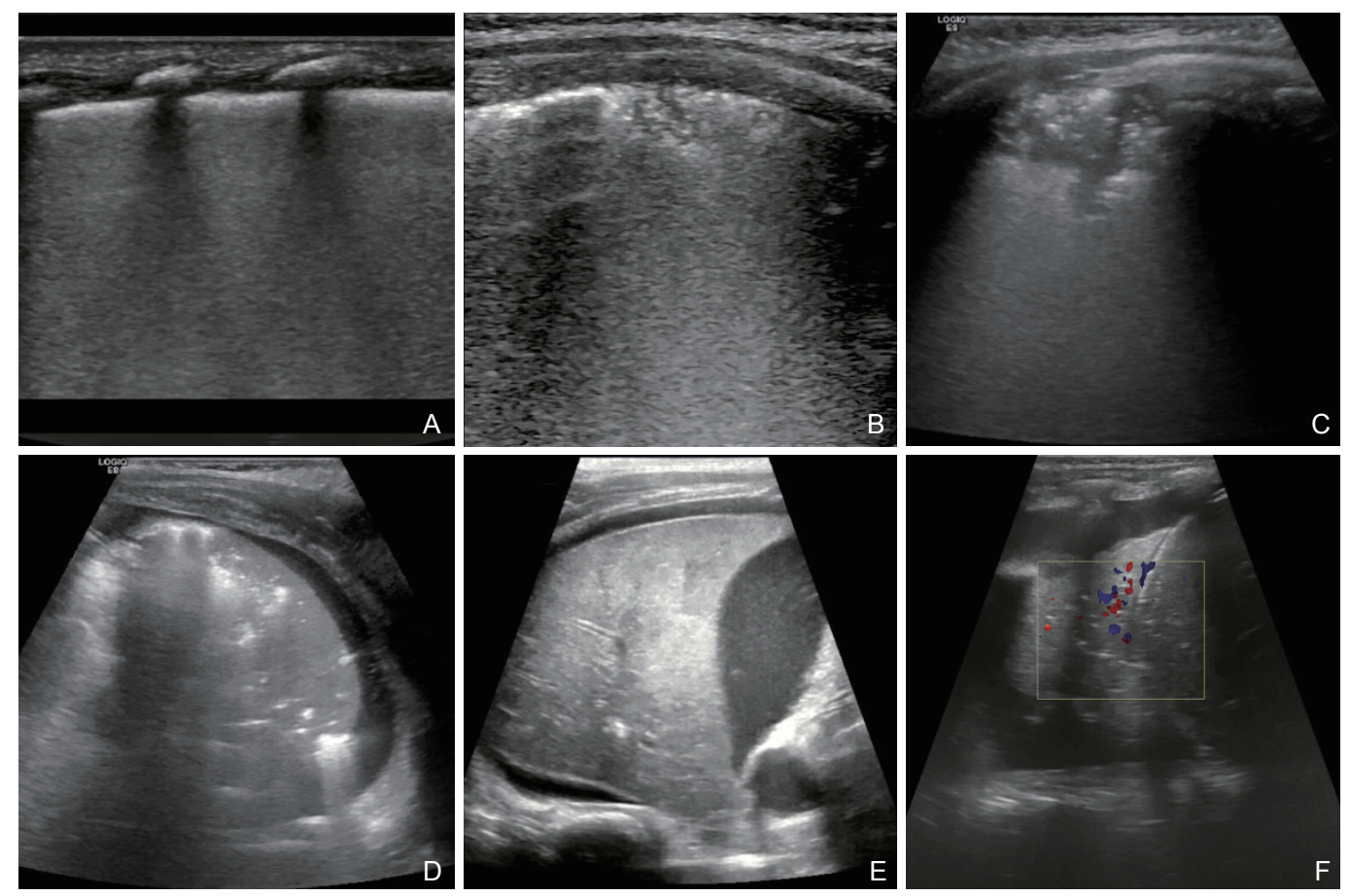

Figure 9 Pulmonary contusion and laceration sonograms. (A) Fusion B-line; (B C) Patchy consolidation; (D) Massive consolidation with B-line of peripheral lung tissue; (E) Pulmonary consolidation with massive pleural effusion; (F) There was blood flow signal in CDFI.

\section{Congenital pulmonary airway malformation (Level C III)}

The pathological mechanism of congenital pulmonary airway malformation is the occlusion of bronchial lumen by hamartomatous tissue or stenosis during embryonic development. There are 5 main types of congenital pulmonary airway malformation: type 0 (alveolar dysplasia or underdevelopment), type 1 (large 
cyst), type 2 (medium cyst), type 3 (mini cyst)), type 4 (peripheral alveolar cyst). All cysts are not connected to the bronchus [45]. Types 1, 2, and 4 are cystic with ultrasound showing cystic lesions in different sizes. Type 1 can cause spontaneous pneumothorax. Type 3 is similar to substantive, with ultrasound showing a homogeneous iso-hyperechoic mass [46].

\section{Pulmonary sequestration (Level C III)}

Pulmonary sequestration is a non-functioning lung tissue formed by the partial ectopic separation of the lung buds from the bronchial tree during the embryonic development. The lung tissue is not connected to the bronchus, and the arterial supplies come from the thoracic or abdominal aorta. Ultrasound appears as a fusiform or triangular-shaped mass in the pleural cavity with bronchial fluid. Color Doppler shows blood flow signal. Pulse wave Doppler shows high-speed and high-resistance blood flow, suggesting that blood flow originated from the systemic circulation [47]. Affected by the gas in the adjacent lung, it is often difficult to visualize the origin of the artery.

\section{Pleuropulmonary blastoma (Level C III)}

Pleuropulmonary blastoma usually occurs in infants. There are three types: purely cystic, cystic and solid, and purely solid [48], presenting monocystic or thinly divided polycystic, cystic solid, and solid mass, respectively $[45,49,50]$. When the tumor involves the pleura, pleural thickening and pleural effusion will be shown. Besides, tumor compression leads to atelectasis, and big tumors can further cause mediastinal displacement.

\section{Extra-corporeal membrane oxygenation (Level A I)}

Extra-corporeal membrane oxygenation (ECMO) can be used for cardiopulmonary support in patients with severe heart failure or respiratory failure. Patients often need a comprehensive ultrasound evaluation of the heart and lungs. The contents of lung ultrasound for patients with ECMO including the assessment of pulmonary edema, pulmonary consolidation, atelectasis, pleural effusion, pneumothorax, hemothorax, etc., and their outcomes [51,52].

\section{Records and Reports}

The pediatric lung ultrasound reports should describe normal and abnormal imaging findings (location, size, range), e.g., the presence and degree of A-lines and B-lines, consolidation location, size, lung sliding, and changes in pleural cavity and mediastinum and its Doppler results. All results and their interpretations should be recorded in the PACS files.

\section{Limitation}

In lung ultrasound, the B-lines from the lung surface are caused by changes in the sub-pleural pulmonary interstitial tissue and alveolar fluid. When the deep lung tissue lesion is covered by the surface inflatable lung, the sub-pleural pulmonary interstitial tissue cannot be displayed using the ultrasound image [53]. Therefore, lung ultrasound is more suitable for the follow-up of known lung diseases, which can appropriately reduce the radiation exposure of children under X-ray.

In summary, lung ultrasound is useful in the preliminary diagnostic work-up of pediatric patients with suspected lung diseases before more invasive tests are performed. Furthermore, its most important application is the follow-up of patients with a previous diagnosis of cardiopulmonary lesions to assess the extent of disease involvement and identify early complications.

\section{Check and Revision Group}

Lixue Yin, $M D^{c}$, Jiawei Tian, $M D^{d}$, Caibao $H u, M D^{e}$, Jun Duan, $M D^{f}$, Mingxing Xie, $M D^{g}$, Ruifang Zhang, $M D^{h}$, Jingjing Ye, $M D^{i}$, Wenjuan Chen, $M D^{j}$, Fangyun Wang, $M D^{k}$, Chunhua zheng, $M D^{l}$, Hong Luo, $M D^{m}$, Qinghua Liu, $M D^{n}$, Yaqing Chen, $M D^{\circ}$

\section{Expert Group :}

Jingjing Ye, $M D^{i}$, Wenjuan Chen, $M D^{j}$, Fangyun Wang, $M D^{k}$, Chunhua zheng, $M D^{l}$, Hong Luo, $M D^{m}$, Qinghua Liu, $M D^{n}$, Yaqing Chen, $M D^{\circ}$, Xiaojing $M a, M D^{p}$, Mei Jin, $M D^{q}$, Hong Zhou, $M D^{r}$, Yinrong Cheng, MD s. Wensheng Yue, $M D^{t}$, Li Yuan, MD ${ }^{u}$, Xiaowei Peng, $M D^{v}$, Zhou Lin, $M D^{a}$, Shuanglan Jiang, $M D^{w}$, Yinghua Sun, $M D^{p}$, Hong Wen, $M D^{x}$, Jiangfan Jiang, $M D^{y}$, Qiao Wang, $M D^{z}$, Haiwei Cao, $M D^{a a}$, Bailing Liu, MD ${ }^{a b}$, Xiaojuan Ma, $M D^{s}$, Xianlian Su, MD ${ }^{a c}$, Xiaofeng Liu, $M D^{a d}$, Guirong Cai, $M D^{a e}$, Liling Shi, MD ${ }^{a f}$, Feng Xie, $M D^{a g}$, Jun Gao, $M D^{u}$, Dan Wang, $M D^{\text {ah }}$, Xiaokang Chen, $M D^{a i}$, Shuangquan $L u, M D^{a j}$, Shiliang $X u, M D^{a k}$, Cuiming Zhang, $M D^{a l}$, Hongying Wei, MD ${ }^{a m}$, Shuping Yang, $M D^{a n}$, Lin Lei, $M D^{a o}$, Xiaqing Xiang, $M D^{a p}$, Liping Mao, MD ${ }^{a q}$, Hong Gao, MD ${ }^{a r}$, Jiangsui Jiang, $M D^{a s}$, Jingyu Wang, $M D^{a t}$, Zhangqian Zhu, $M D^{a u}$

${ }^{a}$ Department of Ultrasound, Shenzhen Children's Hospital; ${ }^{b}$ Department of Ultrasound, the First Bethune Hospital of Jilin University; ${ }^{c}$ Sichuan Provincial People's Hospital; ${ }^{d}$ The 2nd Affiliated Hospital of Harbin Medical University; e Zhejiang Hospital, ${ }^{f}$ ChinaJapan Friendship Hospital; ${ }^{g}$ Union Hospital, Tongji Medical College of Huazhong University of Science and Technology; ${ }^{h}$ The First Affiliated Hospital of Zhengzhou University; ${ }^{I}$ The Children's Hospital Zhejiang University School of Medicine; ${ }^{j}$ Hunan Provincial People's 
Hospital; ${ }^{k}$ Beijing Children's Hospital; ${ }^{l}$ Children's Hospital, Capital Institute of Pediatrics; ${ }^{m}$ West China Second University Hospital, Sichuan University; ${ }^{\text {"Qilu }}$ Children's Hospital of Shandong University; ${ }^{\circ}$ Xinhua Hospital Affiliated to Shanghai Jiao Tong University School of Medicine; ${ }^{p}$ Children's Hospital of Fudan University; ${ }^{q}$ Chengdu women's \& children's Central Hospital; ${ }^{r}$ The Third People's Hospital of Chengdu; ${ }^{s}$ Chengdu First People's Hospital; ${ }^{t}$ Affiliated Hospital of North Sichuan Medical College; "Wuhan children's Hospital; ${ }^{*}$ Jiangxi Provincial People's Hospital; ${ }^{w}$ Dongguan Children's Hospital; ${ }^{x}$ Huizhou Municiple Central Hospital, ${ }^{y}$ Nanning Maternity and Child Health Hospital; ${ }^{2}$ Children's Hospital Affiliated to Chongqing Medical University; ${ }^{a a}$ Children's Hospital of Kaifeng City; ${ }^{a b}$ Xi'an Children's Hospital; ${ }^{a c}$ The Affiliated Hospital of Putian University; ${ }^{\text {ad }}$ Yulin Children's Hospital; ${ }^{\text {ae }}$ Jinniu Maternity and Child Health Hospital of Chengdu; ${ }^{\text {af }}$ Shanxi Provincial Children's Hospital; ${ }^{a g}$ The Second People's Hospital of Lanzhou City; ${ }^{a h}$ Henan Children's Hospital; ${ }^{a i}$ Xiamen Children's Hospital; ${ }^{a j}$ Children's Hospital of Soochow University; ${ }^{a k}$ Haikou People's Hospital; ${ }^{\text {al }}$ Second Hospital of Shanxi Medical University; ${ }^{a m}$ Baotou Children's Hospital; ${ }^{a n}$ Zhangzhou Municipal Hospital of Fujian Province; ${ }^{a o}$ Taihe Hospital; ${ }^{a p}$ Wuxi Children's Hospital; ${ }^{a q}$ Luoyang Maternal and Child Health Hospital; ${ }^{\text {ar }}$ Kunming Children's Hospital; ${ }^{a s}$ Liuzhou Maternity and Child Healthcare Hospita;

${ }^{a t}$ The First People's Hospital of Longquanyi District, Chengdu; ${ }^{a u}$ Wenzhou People's Hospital

\section{Acknowledgments}

The authors thank Zijian Tang for drawing the illustrations, and also wish to appreciate Dr. Yang Hai from Thomas Jefferson University for revising and editing our manuscript.

\section{Conflicts of Interest}

The authors declare no conflict of interests.

\section{Reference}

[1] Xia B. Ultrasonic diagnosis of children (2nd Edition). People's Health Press 2013. [In Chinese]

[2] The Division of Perinatology, Society of Pediatric, Chinese Medical Association, The Division of Neonatal Ultrasound Society, the Chinese Neonatologist Association, Chinese Medical Doctor Association, et al. Guideline on lung ultrasound to diagnose pulmonary diseases in newborn infants. CJCP 2019; 21: 105-113. [In Chinese]

[3] Echocardiography Group of Society of Ultrasound in Medicine, Chinese Medical Association, Chinese Thoracic Society, Chinese Medical Association, Cardiovascular Imaging Group of Chinese Society of Cardiology, Chinese Medical Association. Lung ultrasound protocol for diagnosis of COVID-19 pneumonia and remote consultation (1st edition). Chin J Med Ultrasound (Electronic
Edition) 2020; 17: 213-225. [In Chinese]

[4] Volpicelli G, Elbarbary M, Blaivas M, Lichtenstein DA, Mathis G, Kirkpatrick AW, et al. International evidence-based recommendations for point-of-care lung ultrasound. Intensive Care Med 2012; 38: 577591.

[5] Buda N, Kosiak W, Radzikowska E, Olszewski R, Jassem E, Grabczak EM, et al. Polish recommendations for lung ultrasound in internal medicine (POLLUS-IM). J Ultrason 2018; 18:198-206.

[6] Soldati G, Testa A, Sher S, Pignataro G, La Sala M, Silveri NG. Occult traumatic pneumothorax: diagnostic accuracy of lung ultrasonography in the emergency department. Chest 2008; 133: 204211.

[7] Lichtenstein DA, Mezière GA. Relevance of lung ultrasound in the diagnosis of acute respiratory failure: the BLUE protocol. Chest 2008; 134: 117-125.

[8] Reissig A, Copetti R, Mathis G, Mempel C, Schuler A, Zechner P, et al. Lung ultrasound in the diagnosis and follow-up of communityacquired pneumonia: a prospective, multicenter, diagnostic accuracy study. Chest 2012; 142: 965-972.

[9] Mittal AK, Gupta N. Intraoperative lung ultrasound: A clinicodynamic perspective. J Anaesthesiol Clin Pharmacol 2016; 32: $288-297$.

[10] Lesser TG. Significance of thoracic and lung ultrasound in thoracic surgery. Ultraschall Med 2017; 38: 592-610.

[11] Findeisen H, Trenker C, Figiel J, Greene BH, Görg K, Görg C. Vascularization of primary, peripheral lung carcinoma in CEUS - a retrospective study ( $n=89$ Patients). Ultraschall Med 2019; 40: 603608.

[12] Sperandeo M, Del Colle A, Frongillo E, Rea G, Dimitri L, Cipriani $\mathrm{C}$, et al. Safety maximization of percutaneous transthoracic needle biopsy with ultrasound guide in subpleural lesions in the evaluation of pulmonary consolidation. Respir Res 2019; 20: 68 .

[13] Lichtenstein D. Novel approaches to ultrasonography of the lung and pleural space: where are we now? Breathe 2017; 13:100-111.

[14] Soldati G, Smargiassi A, Mariani AA, Inchingolo R. Novel aspects in diagnostic approach to respiratory patients: is it the time for a new semiotics? Multidiscip Respir Med 2017; 12: 15.

[15] Görg C, Bert T, Görg K. Contrast-enhanced sonography for differential diagnosis of pleurisy and focal pleural lesions of unknown cause. Chest 2005; 128: 3894-3899.

[16] Görg C. Transcutaneous contrast-enhanced sonography of pleuralbased pulmonary lesions. Eur J Radiol. 2007; 64: 213-221.

[17] Di Vece F, Tombesi P, Ermili F, Sartori S. Contrast-enhanced ultrasound (CEUS) and CEUS-guided biopsy in the diagnosis of lung abscess in a patient with achalasia: case report. Interv Med Appl Sci 2013; 5:31-33.

[18] Sperandeo M, Trovato FM, Dimitri L, Catalano D, Simeone A, Martines GF, et al. Lung transthoracic ultrasound elastography imaging and guided biopsies of subpleural cancer: a preliminary report. Acta Radiol 2015; 56: 798-805.

[19] Wei H, Lu Y, Ji Q, Zhou H, Zhou X. The application of conventional us and transthoracic ultrasound elastography in evaluating peripheral pulmonary lesions. Exp Ther Med 2018; 16: 1203-1208.

[20] [Havelock T, Teoh R, Laws D, Gleeson F, Group BTSPDG. Pleural procedures and thoracic ultrasound: British Thoracic Society Pleural Disease Guideline 2010. Thorax 2010; 65 Suppl 2: ii61-76.

[21] European Federation of Societies for Ultrasound in Medicine and Biology (EFSUMB). Minimum training requirements for the practice of medical ultrasound in Europe. Appendix 11: thoracic ultrasound. Ultraschall Med 2009, 30: 90-91.

[22] Skaarup SH, Laursen CB, Bjerrum AS, Hilberg O. Objective and structured assessment of lung ultrasound competence. A 
Multispecialty Delphi Consensus and Construct Validity Study. Ann Am Thorac Soc 2017; 14: 555-560.

[23] Kraft C, Lasure B, Sharon M, Patel P, Minardi J. Pediatric lung abscess: immediate diagnosis by point-of-care ultrasound. Pediatric Emergency Care. 2018; 34: 447-9.

[24] Trenker C, Dohse M, Ramaswamy A, Michel C, Gorg C. Histological validation of pulmonary infarction detected with contrast-enhanced ultrasound in patients with negative computed tomography pulmonary angiogram: A case series. J Clin Ultrasound. 2019; 47: 461-5.

[25] Platz E, Lewis EF, Uno H, Peck J, Pivetta E, Merz AA, et al. Detection and prognostic value of pulmonary congestion by lung ultrasound in ambulatory heart failure patients. Eur Heart J 2016; 37 : 1244-1251.

[26] Dwyer KH, Merz AA, Lewis EF, Claggett BL, Crousillat DR, Lau ES, et al. Pulmonary congestion by lung ultrasound in ambulatory patients with heart failure with reduced or preserved ejection fraction and hypertension. J Card Fail 2018; 24: 219-226.

[27] Pivetta E, Goffi A, Lupia E, Tizzani M, Porrino G, Ferreri E, et al. Lung ultrasound-implemented diagnosis of acute decompensated heart failure in the ED: A SIMEU Multicenter Study. Chest 2015; 148: 202-210.

[28] Pivetta E, Goffi A, Nazerian P, Castagno D, Tozzetti C, Tizzani P, et al. Lung ultrasound integrated with clinical assessment for the diagnosis of acute decompensated heart failure in the emergency department: a randomized controlled trial. Eur J Heart Fail 2019; 21: 754-766.

[29] Liteplo AS, Marill KA, Villen T, Miller RM, Murray AF, Croft PE, et al. Emergency thoracic ultrasound in the differentiation of the etiology of shortness of breath (ETUDES): sonographic B-lines and $\mathrm{N}$-terminal pro-brain-type natriuretic peptide in diagnosing congestive heart failure. Acad Emerg Med 2009; 16: 201-210.

[30] Lichtenstein D, Mézière G, Biderman P, Gepner A, Barré O. The comet-tail artifact. An ultrasound sign of alveolar-interstitial syndrome. Am. J. Respir. Crit. Care Med 1997; 156: 1640-1646.

[31] Picano E, Frassi F, Agricola E, Gligorova S, Gargani L, Mottola G. Ultrasound lung comets: a clinically useful sign of extravascular lung water. JAm Soc Echocardiogr 2006; 19: 356-363.

[32] Picano E, Pellikka PA. Ultrasound of extravascular lung water: a new standard for pulmonary congestion. Eur Heart $J$ 2016; 37: 20972104.

[33] Yang PC, Luh KT, Chang DB, Wu HD, Yu CJ, Kuo SH. Value of sonography in determining the nature of pleural effusion: analysis of 320 cases. Am J Roentgenol 1992; 159: 29-33.

[34] Qureshi NR, Rahman NM, Gleeson FV. Thoracic ultrasound in the diagnosis of malignant pleural effusion. Thorax 2009; 64: 139-143.

[35] Pagano A, Numis FG, Visone G, Pirozzi C, Masarone M, Olibet M, et al. Lung ultrasound for diagnosis of pneumonia in emergency department. Intern Emerg Med 2015; 10: 851-854.

[36] Sferrazza Papa GF, Mondoni M, Volpicelli G, Carlucci P, Di Marco F, Parazzini EM, et al. Point-of-care lung sonography: an audit of 1150 examinations. J Ultrasound Med 2017; 36: 1687-1692.

[37] Nazerian P, Cerini G, Vanni S, Gigli C, Zanobetti M, Bartolucci M, et al. Diagnostic accuracy of lung ultrasonography combined with procalcitonin for the diagnosis of pneumonia: a pilot study. Crit Ultrasound J. 2016; 8: 17.

[38] Reynolds JH, McDonald G, Alton H, Gordon SB. Pneumonia in the immunocompetent patient. Br J Radiol 2010; 83: 998-1009.

[39] Via G, Lichtenstein D, Mojoli F, Rodi G, Neri L, Storti E, et al. Whole lung lavage: a unique model for ultrasound assessment of lung aeration changes. Intensive Care Med 2010; 36: 999-1007.

[40] Karabinis A, Saranteas T, Karakitsos D, Lichtenstein D, Poularas J, Yang $\mathrm{C}$, et al. The 'cardiac-lung mass' artifact: an echocardiographic sign of lung atelectasis and/or pleural effusion. Crit Care 2008; 12: R122.

[41] Cattarossi L. Lung ultrasound: its role in neonatology and pediatrics. Early Hum Dev 2013; 89: S17-19.

[42] Piccolo CL, Ianniello S, Trinci M, Galluzzo M, Tonerini M, Zeccolini $\mathrm{M}$, et al. Diagnostic imaging in pediatric thoracic trauma. Radiol Med 2017; 122: 850-865.

[43] Friedman LM, Tsung JW. Extending the focused assessment with sonography for trauma examination in children. Clin Pediatr Emerg Med 2011; 12: 2-17.

[44] Istasy V, Thompson D, Belisle SS, Gharib M, Lim R. The role of point of care ultrasound in the injured paediatric patient. Curr Pediatr Rev 2018; 14: 41-47.

[45] Leblanc C, Baron M, Desselas E, Phan MH, Rybak A, Thouvenin G, et al. Congenital pulmonary airway malformations: state-of-the-art review for pediatrician's use. Eur J Pediatr 2017; 176: 1559-1571.

[46] Kreiger PA, Ruchelli ED, Mahboubi S, Hedrick H, Scott AN, Russo PA. Fetal pulmonary malformations: defining histopathology. Am. J. Surg. Pathol 2006; 30: 643-649.

[47] Büyükkaya R, Büyükkaya A, Ilce Z, Erdoğmuş B, Küçük A, Özturk B. Role and importance of doppler ultrasound in the diagnosis of pulmonary sequestration: report of two adolescent cases. Med Ultrason 2013, 15: 326-329.

[48] Dehner LP, Watterson J, Priest J. Pleuropulmonary blastoma. A unique intrathoracic pulmonary neoplasm of childhood. Pediatr. Dev. Pathol 1995; 18: 214-226.

[49] Messinger YH, Stewart DR, Priest JR, Williams GM, Harris AK, Schultz KAP, et al. Pleuropulmonary blastoma: A report on 350 central pathology-confirmed pleuropulmonary blastoma cases by the International Pleuropulmonary Blastoma Registry. Cancer 2015; 121: 276-285.

[50] Knight S, Knight T, Khan A, Murphy AJ. Current management of pleuropulmonary blastoma: a surgical perspective. Children (Basel) 2019; 6(8):86.

[51] Del Rey Hurtado de Mendoza B, Sanchez-de-Toledo J, RodríguezFanjul J. Chest ultrasound for guiding lung recruitment maneuvers. Med Intensiva 2018, 42: e20.

[52] Rodríguez-Fanjul Javier,Moreno Hernando Julio,Sánchez-de-Toledo Joan. Lung ultrasound for cardiogenic shock in VA-ECMO. Rev Esp Cardiol (Engl Ed) 2018; 71(5): 393.

[53] American College of Emergency Physicians. Emergency ultrasound guidelines. Ann Emerg Med 2009; 53: 550-570. 